\title{
Modelling of $\mathbf{N}_{2} 1 \mathrm{P}$ emission rates in aurora using various cross sections for excitation
}

\author{
M. Ashrafi ${ }^{1}$, B. S. Lanchester ${ }^{1}$, D. Lummerzheim ${ }^{2}$, N. Ivchenko ${ }^{3}$, and O. Jokiaho ${ }^{1}$ \\ ${ }^{1}$ School of Physics and Astronomy, University of Southampton, UK \\ ${ }^{2}$ Geophysical Institute, University of Alaska, Fairbanks, AK, USA \\ ${ }^{3}$ Space and Plasma Physics, School of Electrical Engineering, KTH, Stockholm, Sweden
}

Received: 22 September 2008 - Revised: 9 June 2009 - Accepted: 11 June 2009 - Published: 24 June 2009

\begin{abstract}
Measurements of $\mathrm{N}_{2} 1 \mathrm{P}$ auroral emissions from the $(4,1)$ and $(5,2)$ bands have been made at high temporal and spatial resolution in the region of the magnetic zenith. The instrument used was the auroral imager ASK, situated at Ramfjordmoen, Norway (69.6 N, 19.2 E) on 22 October 2006. Measurements from the European Incoherent Scatter Radar (EISCAT) have been combined with the optical measurements, and incorporated into an ionospheric model to obtain height profiles of electron density and emission rates of the $\mathrm{N}_{2} 1 \mathrm{P}$ bands. The radar data provide essential verification that the energy flux used in the model is correct. One of the most important inputs to the model is the cross section for excitation to the $\mathrm{B}^{3} \Pi_{\mathrm{g}}$ electronic state, as well as the cross sections to higher states from which cascading into the B state occurs. The balance equations for production and loss of the populations of all levels in each state are solved in order to find the cascade contributions. Several sets of cross sections have been considered, and selected cross sections have been used to construct "emission" cross sections for the observed bands. The resulting brightnesses are compared with those measured by ASK. The importance of specific contributions from cascading is found, with more than $50 \%$ of the total brightness resulting from cascading. The cross sections used are found to produce a range of brightnesses well within the uncertainty of both the modelled and measured values.
\end{abstract}

Keywords. Atmospheric composition and structure (Ion chemistry of the atmosphere) - Ionosphere (Auroral ionosphere) - Space plasma physics (Ionization processes)

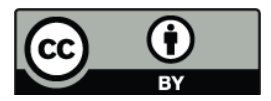

Correspondence to: M. Ashrafi (m.ashrafi@soton.ac.uk)

\section{Introduction}

Molecular nitrogen is the major constituent in the atmosphere up to $200 \mathrm{~km}$ altitude. Hence cross sections for electron collisions with nitrogen are of particular importance in explaining the natural phenomenon of the aurora, as well as other phenomena such as airglow and lightning. The $\mathrm{N}_{2}$ first positive band from its triplet system is one of the strongest nitrogen emissions in the auroral spectrum with band intensity of about $900 \mathrm{kR}$ (Gattinger and Vallance-Jones, 1974). Bands of the first positive system of molecular nitrogen are produced by transitions from the vibrational states of the $B^{3} \Pi_{g}$ electronic state to the vibrational states of the $\mathrm{A}^{3} \Sigma_{\mathrm{u}}^{+}$electronic state. Cross sections are measured by two fundamentally different methods: either by observing the energy degraded electrons that result from the collision, or by observing the photons resulting from the collision. The first method measures the excitation cross section, the second the emission cross section, from which an excitation cross section is estimated. There are many publications on determining the electron impact cross sections of the $\mathrm{N}_{2}$ molecule, both from laboratory measurements and using theoretical approaches. Reviews have been published by several authors (see for example Majeed and Strickland, 1997; Brunger and Buckman, 2002; and Itikawa, 2005).

Experimental methods of deriving cross sections rely on accurate measurements of the vibrational population rates which largely depend on optical transition probabilities of the entire band system. A method commonly used is to pass an electron beam of a given energy through the molecular gas and measure the absolute intensity of the radiation. Stanton and St. John (1969) measured optical cross sections for all the $\mathrm{N}_{2} 1 \mathrm{P}$ bands, and determined the apparent excitation cross section of the B state, demonstrating the importance of the cascade contribution from the $\mathrm{C}$ to $\mathrm{B}$ state. Shemansky and Broadfoot (1971) determined the $\mathrm{N}_{2} 1 \mathrm{P}$ excitation cross

Published by Copernicus Publications on behalf of the European Geosciences Union. 
section by measuring one or two bands of the system relative to the $\mathrm{N}_{2}^{+} 1 \mathrm{~N}(0,0)$ band. The total apparent cross section was calculated using estimated population rates. Their result was a factor of two larger than that of Stanton and St. John (1969). For the alternative method, using scattering of electrons, Cartwright et al. (1977a,b) used electron energy loss measurements to obtain excitation cross sections of $\mathrm{N}_{2}$ electronic states by numerical integration of the differential cross sections over a range of angles $5-138^{\circ}$. Similar experimental measurements were made by Brunger and Teubner (1990), whose result for excitation to the B state is up to three orders of magnitude higher than that of Cartwright et al. (1977a). The large discrepancies between results makes the choice of cross sections used in modelling the $\mathrm{N}_{2} 1 \mathrm{P}$ brightness a critical but difficult matter.

Excitation cross sections of $\mathrm{N}_{2}$ states have also been obtained theoretically by application of quantummechanical approximations, such as the Ochtur-Rudge approach (Cartwright, 1970) and Born-Ochtur-Rudge using Gaussian type atomic orbitals as wave functions (Chung and Lin, 1972). Gillan et al. (1996) calculated the integral cross sections for nitrogen using the R-matrix method which is most reliable for low-energy excitations. Tashiro and Morokuma (2007) repeated the R-matrix theory for deriving the low energy electron impact excitation, the results of which are in good agreement with Cartwright et al. (1977a).

The role of cascade contributions to the triplet nitrogen system has been the subject of many further publications. Cartwright et al. (1971) investigated the cascade contributions to the vibrational population of the $\mathrm{N}_{2}$ first positive system by applying a mixed set of experimental and theoretical data for electron impact excitation cross sections. These authors indicated the importance of intersystem cascade processes $(\mathrm{B} \rightleftharpoons \mathrm{W})$ and $(\mathrm{B} \rightleftharpoons \mathrm{A})$ following the work of $\mathrm{Wu}$ and Benesch (1968) and Gilmore (1969). Their results were challenged by Shemansky and Broadfoot (1973) mainly with respect to the significance of specific cascade contributions. Using the best available electron impact cross sections at the time, Cartwright (1978) developed a set of complete vibrational populations of the $\mathrm{N}_{2}$ band system taking into account intersystem cascading and vibrationally dependent quenching. Morrill and Benesch (1996) also studied the $\mathrm{N}_{2}$ triplet state vibrational population, taking into account additional collisional processes, in particular the intersystem collisional transfer of excitation (ICT) between the B state and the A, W and $B^{\prime}$ states, resulting from collisions with ground state $\mathrm{N}_{2}$. Their improved result of the B state population is in good agreement with that of Cartwright (1978), except at very low heights $(\sim 80-90 \mathrm{~km})$. Their result indicated population enhancement of higher vibrational levels due to the effects of ICT and metastable states, which would produce auroral red lower borders at these heights.

There have been attempts to determine the best values of the $\mathrm{N}_{2}$ excitation cross section by using all available experimental and theoretical data (e.g. Brunger et al., 2003). Fol- lowing this work, Itikawa (2005) compiled cross section data for electron collisions with nitrogen molecules based on 104 references dating to 2003. For most of the lower states, theoretical cross sections derived by R-matrix calculations were used to obtain the detailed structure near the threshold. A weighted average of the experimental cross section was used for deducing cross sections for higher energies. Tables of recommended values are provided of cross sections for electron impact excitation to all electronic states of $\mathrm{N}_{2}$. These have been used as one of the inputs in the present work.

The brightness of specific optical emissions in the aurora depends on the density of the atmospheric constituents and the spectrum of the precipitating electrons as well as the scattering and emission cross sections of various species. In this paper we compare measured emission from the $\mathrm{N}_{2}$ first positive band system made with a new auroral imager ASK (Auroral Structure and Kinetics) with model results using selected cross sections. A combination of optical and radar data has been used to produce the input to the model. The output is the time history of the brightness of two bands of the molecular nitrogen emission during auroral precipitation events. By comparing the model results with measured brightnesses, we assess various cross sections for excitation of the $\mathrm{N}_{2}$ molecule, and highlight the importance of estimating accurately the contribution to the emissions from cascading into the B state.

\section{Instrumentation}

The ASK instrument has three co-aligned imagers (ASK1, ASK2 and ASK3), each equipped with an EMCCD detector with $512 \times 512$ pixel chip. Image rates of 32 frames per second are achievable. Each imager is fitted with a removable Galilean converter, which provides a $150 \mathrm{~mm}, \mathrm{f} / 1.0$ lens. With the converters in position, the field of view of each camera is $3^{\circ} \times 3^{\circ}$ which is equivalent to $5 \times 5 \mathrm{~km}$ at $100 \mathrm{~km}$ height. Narrow band filters are selected for each optical system. In the present work the ASK1 filter had a central wavelength of $673.0 \mathrm{~nm}$, with width containing the vibrational band emissions from transitions $(4,1)$ and $(5,2)$ of the $\mathrm{N}_{2} 1 \mathrm{P}$ from the $\mathrm{B}^{3} \Pi_{g}$ state to the $\mathrm{A}^{3} \Sigma_{\mathrm{u}}^{+}$state. The ASK2 filter is designed to measure emission from the oxygen ion at $732.0 \mathrm{~nm}$, caused by the $\mathrm{O}^{+}\left({ }^{2} \mathrm{P}-{ }^{2} \mathrm{D}\right)$ transition. The ASK3 filter measured $777.4 \mathrm{~nm}$ from atomic oxygen. Only ASK1 data are used in this paper, binned in $2 \times 2$ pixels.

Height profiles of electron density in the ionosphere are obtained with the EISCAT radar situated near Troms $\varnothing$, Norway $(69.6 \mathrm{~N}, 19.2 \mathrm{E})$. The present observations were made with the UHF antenna, pointed along the local magnetic field line. The radar programme "arc1" was used, which provides $0.44 \mathrm{~s}$ time resolution with range resolution of about $900 \mathrm{~m}$ between $96 \mathrm{~km}$ and $422 \mathrm{~km}$. The electron density height profiles are used to estimate the time varying energy spectra used as input for the modelling. 


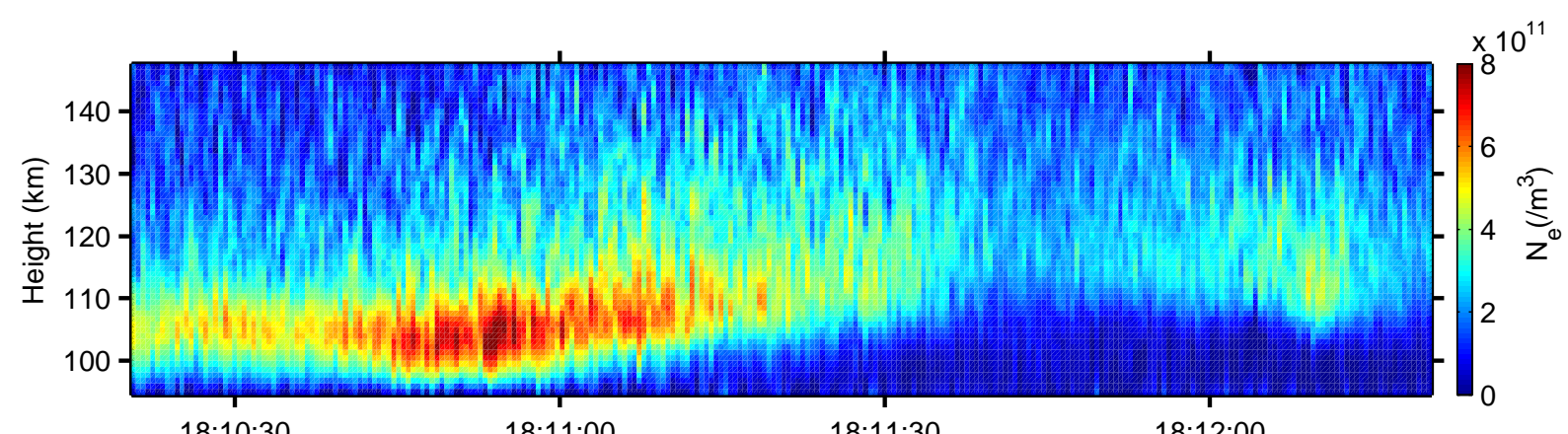

$18: 10: 30$

18:11:00

18:11:30

$18: 12: 00$

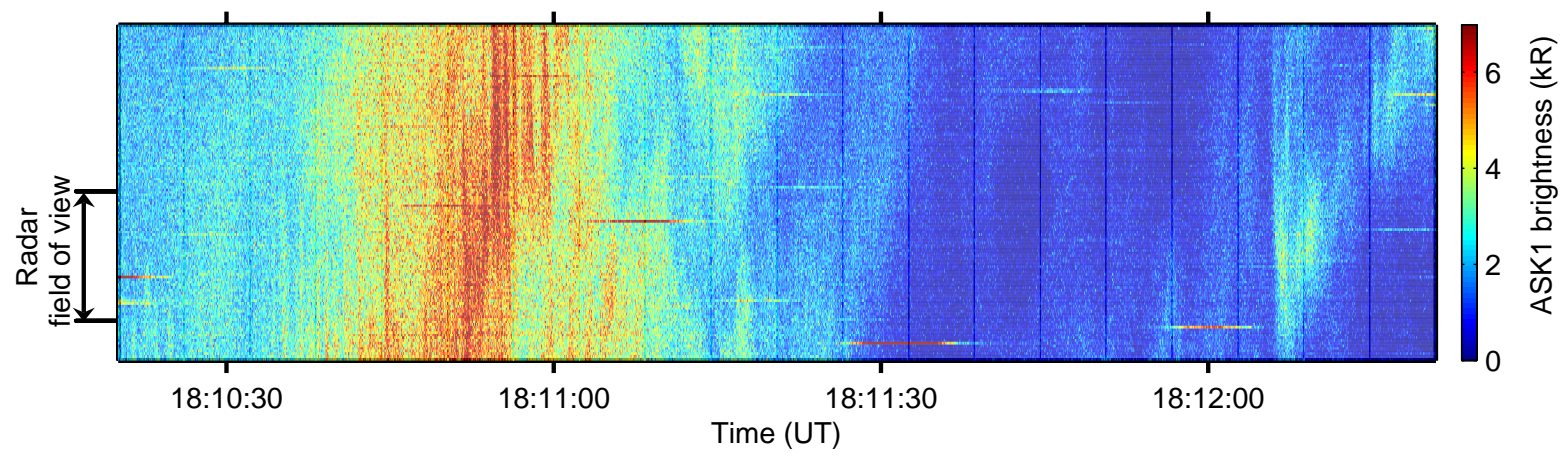

Fig. 1. Top: Electron density profiles in the E region measured with the EISCAT UHF radar. Bottom: Time series of slices through the ASK1 images in the N-S meridian.

\section{Modelling}

The auroral model used in this work is time-dependent and solves the electron transport equation (Lummerzheim and Lilensten, 1994) at timesteps chosen to match the auroral observations and conditions, usually at sub-second resolution for active aurora. It uses a one-dimensional height grid ranging from $85 \mathrm{~km}$ to $500 \mathrm{~km}$. The grid step size increases with the atmospheric scale height. The model requires a neutral atmosphere as input, for which the MSIS90 thermospheric model (Hedin, 1991) was used for appropriate conditions on the date of the observations. Resulting height profiles of auroral ionisation, excitation and electron heating rates are used as input to the ion chemistry part of the model, which solves the coupled continuity equations for all important positive ions and minor neutral species, and the electron and ion energy equations. The final output of the model is a time series of height profiles of emission rates, densities and temperatures. These can be integrated in height to give a time series of changes to these parameters in the magnetic zenith direction.

One of the main inputs to the electron transport model are electron impact cross sections of the major atmospheric neutral constituents. Excitation cross sections and the energy losses of each individual excited state are required to calculate the energy degradation at each step of the electron transport. The cross sections used here are combined into sets. The first cross section set is taken from the work of Lum- merzheim (1987) and is referred to as Set 1 from now on. A similar cross section set provided by R. Link (private communication) is referred to as Set 2 . For the transport and energy degradation calculations, Set 1 was used. As discussed in Lummerzheim and Lilensten (1994), changing a single excitation cross section has a very small effect on the transport calculation, which is dominated by the large energy losses and large cross section for ionisation. However, this is not the case for the calculation of emission rates resulting from the precipitating input spectra. Careful selection of individual cross sections for the relevant excited states is required. The variation in model results using different cross sections for calculating emission rates is the subject of this work. Three sets of excitation cross sections are used in the modelling of optical emissions: Set 1 and 2 as referred to above, and a set obtained from the review by Itikawa (2005) referred to as Set 3 .

The other main input is a set of time varying energy spectra, which can be specified by the electron energy flux, and a spectral shape, which is defined by peak values of Maxwellian and Gaussian distributions, either separately or combined (Lanchester et al., 1997). The incident electron spectra were determined by an iterative inversion of the density profiles from EISCAT combined with measured auroral brightnesses. This detailed process is the subject of another paper which uses modelling to study the small scale auroral changes in all three ASK cameras during the same events described here (Lanchester et al., 2009). 




Fig. 2. Sequence of images from ASK1 camera showing filamentation in active aurora in nitrogen emission. The circles mark the radar field of view. The lines mark the north-south meridian slice used in Fig. 1.
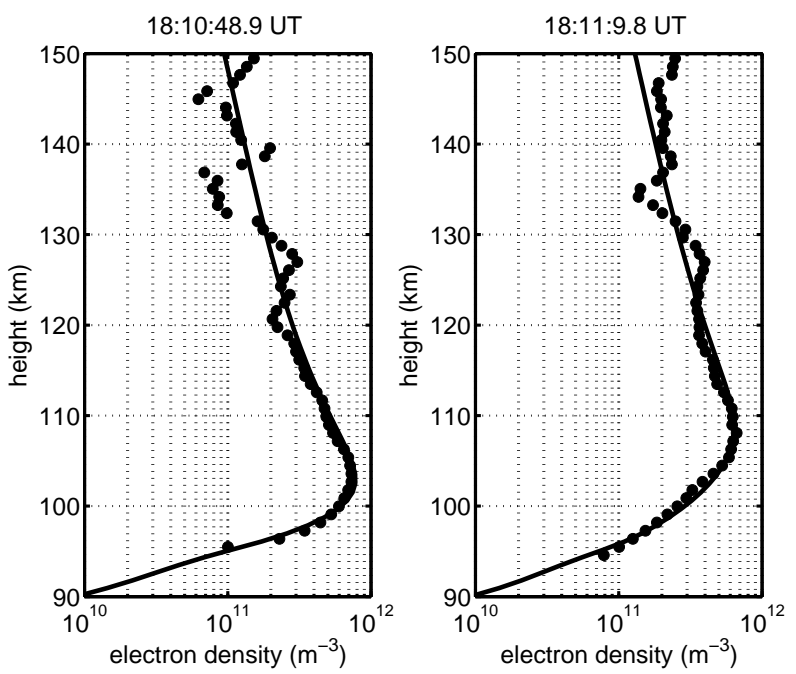

Fig. 3. Modelled electron density profiles (solid line) compared with smoothed electron density profiles (circles) measured by the EISCAT UHF radar.

\section{Analysis: measured and modelled emissions}

The data used for this analysis were obtained on 26 October 2006 in a two-minute interval between 18:10:15 UT and 18:12:15 UT. An overview of the events is shown in Fig. 1. The top panel is EISCAT electron density height profiles in the $\mathrm{E}$ region, showing that precipitation increased to a maximum intensity and penetrated to heights of $100 \mathrm{~km}$ at about 18:10:50 UT. After this time the density reduced and the peak density increased in height. A further burst of precipitation followed at 18:12:10 UT. The second panel of Fig. 1 is a time series of meridian (magnetic north-south) slices of 1 binned pixel width across the $3^{\circ}$ field of view of the ASK1 images. The position of the radar field of view $\left(0.6^{\circ}\right.$ half-width $)$ is marked on the ordinate.

This way of displaying the data gives a summary of events; it can be seen that within the time of increased emissions there is much structure, both temporal and spatial. Figure 2 is a series of sample ASK1 images of the auroral filamentation at the time of maximum intensity. The images are separated by $0.1 \mathrm{~s}$, showing the fast variations occurring. The position

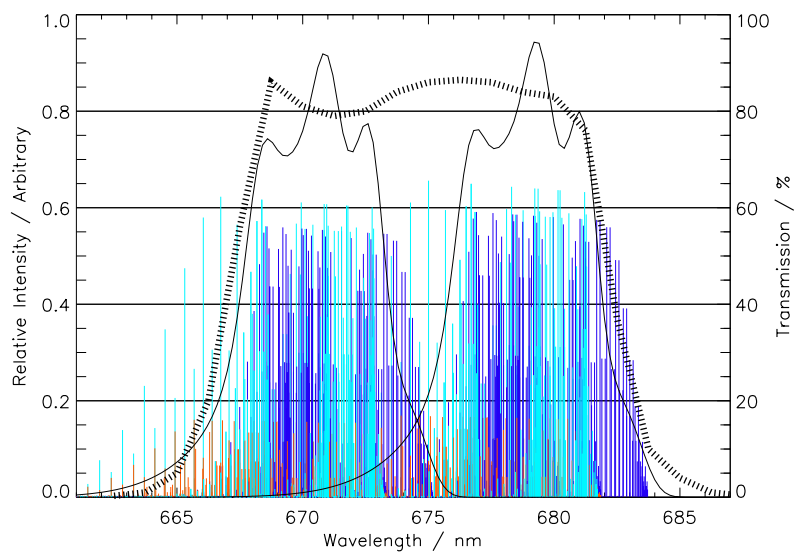

Fig. 4. Relative intensities of synthetic spectra of $\mathrm{N}_{2}$ first positive $(5,2)$ and $(4,1)$ bands, with the former on the left. The rotational lines are convolved with an instrument function of $0.5 \mathrm{~nm}$ for a rotational temperature of $300 \mathrm{~K}$ (thin black lines). The ASK1 $673.0 \mathrm{~nm}$ filter transmission curve is superimposed (dashed line).

of the radar field of view and the meridian slice (width corresponding to $20 \mathrm{~m}$ at $100 \mathrm{~km}$ height) are superimposed on the images. In the following, the ASK1 brightness in the radar field of view (as drawn) is referred to as $I_{\text {obs }}$.

The radar height profiles of electron density are used as a starting point to provide input energy spectra for the model, giving an estimate of both the total energy flux and the shape of the energy spectrum at each time step. The resulting output ion densities are summed to give height profiles of electron density, and these are compared with the radar measurements of E-region electron density for the entire interval. An iterative process is used to improve the fit, and hence confirm the input values for the energy spectrum. The values of flux are particularly important in this respect. Figure 3 shows two examples of electron density profiles in the E-region, measured and modelled. The first example is at the time of the maximum intensity at 18:10:49 UT with a clear peak fitted at about $103 \mathrm{~km}$. The second example, $20 \mathrm{~s}$ later, is when the aurora was changing in nature from dynamic narrow filaments to less discrete flickering patches. The peak density has reduced and the height of the peak has risen to $108 \mathrm{~km}$. In both examples the model fit is excellent, as is the case for the entire run. As a result, the input flux is considered to be an accurate estimate, giving weight to the model results for the $\mathrm{N}_{2} 1 \mathrm{P}$ emissions.

The ASK1 filter, centred on $673.0 \mathrm{~nm}$, contains the vibrational band emissions from transitions $(4,1)$ and $(5,2)$ of $\mathrm{N}_{2}$ 1P. In order to estimate the fraction of the emissions from the two bands which pass through the filter, synthetic spectra are produced following the method adapted from Jokiaho et al. (2008) as shown in Fig. 4. The dark blue, red, and light blue lines are from the $\mathrm{P}, \mathrm{R}$ and $\mathrm{Q}$ branches respectively, with the $(5,1)$ band on the left. These lines have been convolved with an instrument function of $0.5 \mathrm{~nm}$ to give the thin black 


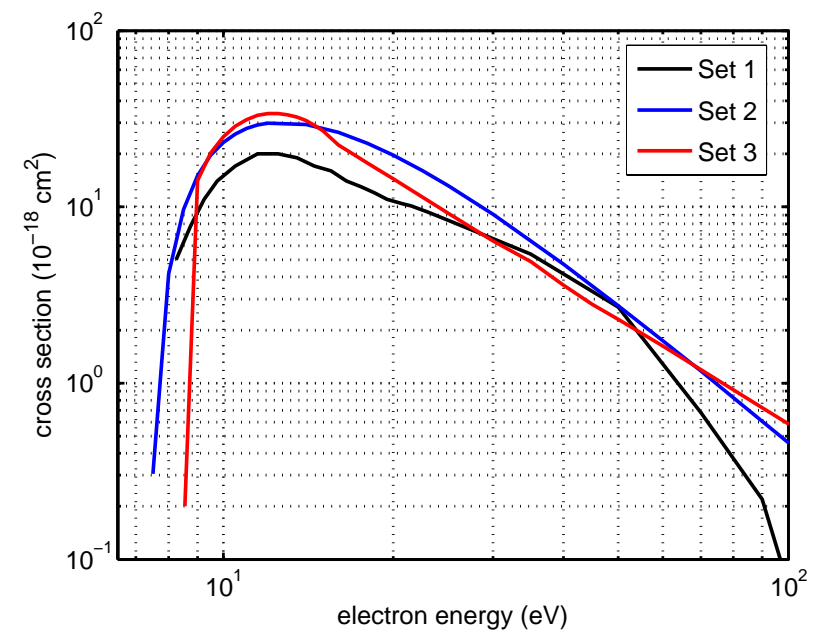

Fig. 5. Comparison between values of electron impact cross sections of $\mathrm{N}_{2} \mathrm{~B}^{3} \Pi$ state for three cross section sets.

lines. The shape of the filter transmission is shown superimposed on the two bands as the dashed line. A transmission factor $(T)$ is then obtained by convolving these two curves and integrating. Although the two bands appear almost identical here, their relative intensity depends on the vibrational temperature of the $\mathrm{N}_{2}$ ground state, Franck-Condon factors and Einstein coefficients, including direct excitation and cascading. The population distribution of the ground state of $\mathrm{N}_{2}$ changes very little below a vibrational temperature of $1000 \mathrm{~K}$. The value of $T$ is also affected by the rotational temperature through the shape of each band. The transmittance of the $(4,1)$ band convolved with the filter curve does not vary significantly with temperature. However, modelling shows that the theoretical intensity of the $(5,2)$ band transmitted through the filter varies from 0.74 at $300 \mathrm{~K}$ to 0.63 at $1000 \mathrm{~K}$. Assuming a rotational temperature of $300 \mathrm{~K}$, the filter transmission factor for the two bands is estimated to be 0.76 , decreasing to 0.69 at $1000 \mathrm{~K}$. The former value has been assumed as being more appropriate for the relatively high energy aurora under study.

In order to compare the measured brightness $I_{\mathrm{obs}}$ with model results, the calculated $\mathrm{N}_{2}$ emission rate is integrated in height to give a surface brightness in Rayleighs, $I_{\text {mod }}$. The analysis requires a choice of cross sections for excitation to the relevant states. Excitation cross sections to the B state from Set 1, Set 2 and Set 3 are plotted in Fig. 5. Using these excitation cross sections, the resulting $I_{\text {mod }}$ would contain no contribution from cascading to the B state from other higher states. This cascade contribution can be quantified by constructing effective excitation cross sections, using excitation cross sections for the $\mathrm{A}, \mathrm{B}^{\prime}, \mathrm{C}$ and $\mathrm{W}$ states. All contributions to cascading into $\mathrm{B}$ from all higher states are taken into account, including reverse transitions from high levels of the A state, and transitions out of high levels of $\mathrm{B}$ to $\mathrm{A}, \mathrm{B}^{\prime}$ and $\mathrm{W}$, and back again to lower B levels.

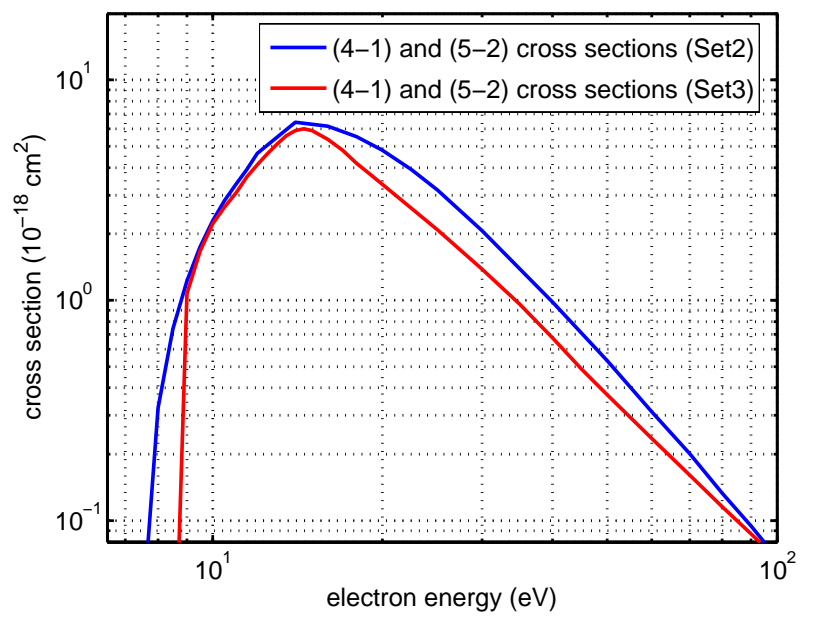

Fig. 6. Constructed emission cross sections for $\mathrm{N}_{2} 1 \mathrm{P}(4,1)$ and $(5,2)$ bands, including all cascades from B, A, C, B', W states (Set 2 and Set 3$)$.

The cascade contributions are calculated by solving the balance equation for steady state

$\mathbf{M}_{i, j} \boldsymbol{n}_{j}=S_{i}$

where $\mathbf{M}_{i, j}$ is the matrix of coefficients containing all production and losses in radiative transitions, the $\boldsymbol{n}_{j}$ are the populations of all the vibrational levels in all states and the $\boldsymbol{S}_{i}$ are the direct electron impact excitation terms for all levels of all states. The latter are the product of the excitation rate for each electronic state, $\eta_{\text {state }}$, and the Franck-Condon factor for the particular level in each state. The emission rate between level $v^{\prime}$ of the B state and $v^{\prime \prime}$ of the A state is given by

$\eta_{v^{\prime}-v^{\prime \prime}}=W_{C} \eta_{C}+W_{B} \eta_{B}+W_{B^{\prime}} \eta_{B^{\prime}}+W_{W} \eta_{W}+W_{A} \eta_{A}$

where the factors $W_{C}, W_{B}, W_{B^{\prime}}, W_{W}$ and $W_{A}$ for each state are obtained from the steady state population of the emission parent level by multiplying by the Einstein coefficient for the transition.

In order to estimate the emissions from the $(4,1)$ and $(5,2)$ bands, effective emission cross sections can be constructed. These are given as

$$
\begin{aligned}
\sigma_{4-1}= & W_{C}(4,1) \sigma_{C}+W_{B}(4,1) \sigma_{B}+W_{B^{\prime}}(4,1) \sigma_{B^{\prime}} \\
& +W_{W}(4,1) \sigma_{W}+W_{A}(4,1) \sigma_{A} \\
\sigma_{5-2}= & W_{C}(5,2) \sigma_{C}+W_{B}(5,2) \sigma_{B}+W_{B^{\prime}}(5,2) \sigma_{B^{\prime}} \\
& +W_{W}(5,2) \sigma_{W}+W_{A}(5,2) \sigma_{A}
\end{aligned}
$$

where $\sigma_{C}$ is the excitation cross section of the transition of the $\mathrm{X}$ state $\left(0\right.$ level) to all levels of the $\mathrm{C}$ state, $\sigma_{B}$ is the excitation cross section of the transition of the $\mathrm{X}$ state (0 level) to all levels of the B state and so on. Values of $W_{C}, W_{B}, W_{B^{\prime}}, W_{W}$ and $W_{A}$ for the two specified bands are 
Table 1. Values of $W_{C}, W_{B}, W_{B^{\prime}}, W_{W}$ and $W_{A}$ for emission cross section of $\mathrm{N}_{2} 1 \mathrm{P}(4,1)$ and $(5,2)$ bands.

\begin{tabular}{ccc}
\hline & $(4,1)$ & $(5,2)$ \\
\hline$W_{C}$ & 0.0127 & 0.0107 \\
$W_{B}$ & 0.0313 & 0.0351 \\
$W_{B^{\prime}}$ & 0.0312 & 0.0411 \\
$W_{W}$ & 0.0292 & 0.0359 \\
$W_{A}$ & 0.0104 & 0.0105 \\
\hline
\end{tabular}

given in Table 1. Values of Franck-Condon factors and transition probabilities are taken from Gilmore et al. (1992).

The combined cross sections for the $(4,1)$ and $(5,2)$ bands using Set 2 and Set 3 are plotted in Fig. 6. After inserting these cross sections into the model and applying the filter transmission factor $T=0.76$, the resulting $I_{\text {mod }}$ is then an estimate of surface brightness from the two bands measured by the ASK instrument. Figure 7 is a summary of the results for $I_{\text {mod }}$ using the two cross sections of Fig. 6. Set 1 cross section is not shown in Fig. 6 nor are the model results using Set 1 shown in Fig. 7. The values are all lower than those for Set 3 by about $25 \%$. The dark solid line is the ASK1 measured brightness, $I_{\mathrm{obs}}$. The modelled brightness from Set 3 (red) makes a very good fit, and that from Set 2 (blue) is about $20 \%$ greater. We note that using only the B state for excitation would lead to an underestimate of the brightness by more than a factor of two.

A more direct approach for modelling the $\mathrm{N}_{2} 1 \mathrm{P}$ band emission rates might be to use measured emission cross sections, so that all cascades are included. The only such cross section curves available to the authors are those of Stanton and St. John (1969) and Shemansky and Broadfoot (1971). The latter measurements are of the $(3,1)$ band and not the $(4,1)$ or $(5,2)$ bands. Therefore a direct comparison cannot be made. Since Shemansky and Broadfoot (1971) compare and discuss their cross section for the $(3,1)$ band with that of Stanton and St. John (1969), we can apply both these cross sections (see Fig. 2 of Shemansky and Broadfoot, 1971) to the model and obtain the emissions from this band. We have then constructed a cross section for this emission as described above using cross sections from Set 3 . The resulting $(3,1)$ band emissions from the constructed cross section are lower than those from the cross sections of Stanton and St. John (1969) by about $20 \%$ and considerably lower than the emissions from the cross sections of Shemansky and Broadfoot (1971). As a further comparison we have taken the measured brightnesses tabulated by Vallance-Jones (1974) in Table 4.12, and estimated a scaling factor for the $(3,1)$ band from the measured ASK emissions of the $(4,1)$ and $(5,2)$ bands. This rough method shows that the emission brightness obtained in this way agrees more closely with the brightness obtained using the cross section of Stanton and
St. John (1969) rather than that of Shemansky and Broadfoot (1971).

\section{Discussion}

To calculate the $\mathrm{N}_{2} 1 \mathrm{P}$ emissions from excitation of the $\mathrm{B}$ state it is necessary to take into account (a) direct excitation (b) cascading from higher energy states and (c) de-excitation by mechanisms other than photon emission (quenching). To calculate brightnesses, the emission is integrated along the line of sight, and therefore a correction for extinction (the sum of scattering and absorption) is required. In some cases it may also be necessary to consider scattering into the line of sight from sources outside the field of view (Gattinger et al., 1991). In the present case, quenching and scattering into the line of sight can be neglected, but extinction is included. Also excluded is the intersystem collisional transfer of excitation between the B state and other states (A, W and $\left.\mathrm{B}^{\prime}\right)$, as studied by Morrill and Benesch (1996). Such collisional processes would be relevant for higher energy aurora (with lower borders below $90 \mathrm{~km}$ ) than is analysed in this event. The present event was chosen to have a lower border above $100 \mathrm{~km}$, in order to make the fitting of electron density profiles more accurate.

In this work, excitation cross sections for all higher energy states known to have a significant cascade contribution have been incorporated. It is possible that there are other cascade contributions which should be included. McConkey and Simpson (1969) mention possible contributions from $\mathrm{C}^{\prime}$ (Goldstein-Kaplan) and D (fourth positive), and Ohmori et al. (1988) discuss cascading from the E state. In the present work we make the assumption that these cascade contributions are of minor importance compared with those that we have included in the model. One important result of the present work is that we are able to determine the relative importance of the included cascades by solving Eq. (1) for the populations of each level of each state. In the case of the $(4,1)$ and $(5,2)$ bands that we measure, the contribution from radiative transfer between upper $B$ levels and other nearby levels was found to be negligible. The largest contributions to cascading are from the $\mathrm{C}$ and $\mathrm{B}^{\prime}$ states.

There are very clear differences between the cross section sets that have been used in this work. Set 1 was formed on the basis of work by Stamnes and Rees (1983), and was later updated and used by Lummerzheim and Lilensten (1994) to compare the modelled ratio of $\mathrm{N}_{2} 2 \mathrm{PG}$ to $\mathrm{N}_{2}^{+}$ emissions with auroral observations. In particular they reduced the excitation cross section for $\mathrm{N}_{2}\left(\mathrm{C}^{3} \Pi\right)$ to agree with that of Cartwright et al. (1977a). Set 2 values are based on cross sections provided by R. Link (private communication), which he renormalised from the review compilation of Trajmar et al. (1983). The cross sections of Set 3 have some common roots, as explained in Itikawa (2005). The cross sections from that paper are adopted from the conclusions of Brunger 


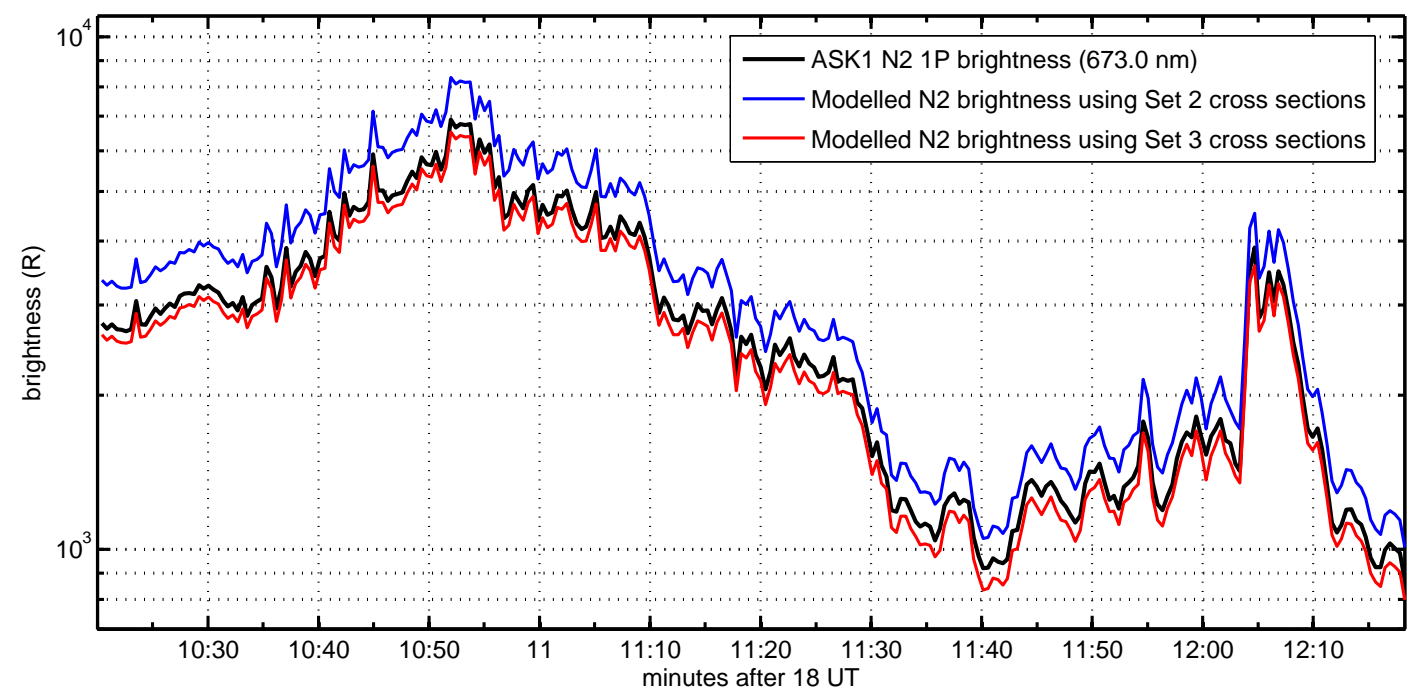

Fig. 7. Comparison between ASK1 $(673.0 \mathrm{~nm})$ measured intensity (black) and modelled $\mathrm{N}_{2} 1 \mathrm{P}$ emission using cross sections from Set 2 (blue) and Set 3 (red).

et al. (2003) who determined the best values based on the results of Trajmar et al. (1983), Campbell et al. (2001), Ohmori et al. (1988) and the theoretical work of Gillan et al. (1996). All the source data for this set of cross sections can be reviewed in Brunger and Buckman (2002). Other sources of cross sections have been considered, e.g. Johnson et al. (2005) which are taken from measurements of Khakoo et al. (2005). The values published are on a coarser energy grid, allowing errors from interpolation. The cross sections and resulting emissions are lower than those used to produce Set 3.

A direct comparison with emission cross sections of Stanton and St. John (1969) and Shemansky and Broadfoot (1971) has not been possible using optical emissions, since their published data are for the $(3,1)$ band. However we have attempted a rough comparison of the model results for these cross sections with constructed cross sections for this band using Set 3. As expected, the cross section of Shemansky and Broadfoot (1971) gives much larger values than that of Stanton and St. John (1969), which agree better with the emissions from the constructed cross section. The factor of two difference between the emission cross sections of Shemansky and Broadfoot (1971) and Stanton and St. John (1969) is discussed by Shemansky and Broadfoot (1971). They suggested that discrepancies between the cross sections published by various authors could be the result of several factors, including the relatively minor effect of experimental calibration of instruments, the comparatively long lifetime of some vibrational levels, and the contribution to the population from $\mathrm{N}_{2}$ 1P low pressure afterglow. However, although only a rough comparison, the present results would appear to support the earlier measurements.

The cross sections that have been applied in the model have uncertainties associated with them. For example,
Brunger et al. (2003) estimated an uncertainty of up to $\pm 35 \%$ for excitation of the $\mathrm{B}^{3} \Pi_{\mathrm{g}}$ and $\mathrm{W}^{3} \Delta_{\mathrm{u}}$ states, $\pm 40 \%$ for $\mathrm{B}^{\prime}{ }^{3} \Sigma_{\mathrm{u}}^{-}$and $\pm 30 \%$ for $\mathrm{C}^{3} \Pi_{\mathrm{u}}$. Uncertainties arising from the transport model have been discussed in Lummerzheim and Lilensten (1994) and estimated to be of the order of 15-20\%. These uncertainties are increased if the atmospheric densities are not well known. In the present work, the radar data provide a check that the model produces consistent results. The agreement between the modelled and measured electron density height profiles as shown in Fig. 3 demonstrates that the values of energy flux and peak energy used in the model are very good estimates for E-region heights. Therefore we consider the over-riding uncertainty in the model is from the cross sections. In these data the radar profiles confirm that the ionisation is almost all in the E region, and the result of precipitation with peak energies between 4-11 keV.

A possible systematic error in measurements from the ASK1 camera can be estimated from the contribution to the background in the wavelength range of the filter. In this case it is relatively free of significant emissions which could contaminate the results. The main contribution comes from bands of the $\mathrm{N}_{2}^{+}$Meinel and $\mathrm{O}_{2}^{+} 1 \mathrm{~N}$. Referring to Fig. 4.5 of Vallance-Jones (1974), the contribution from $\mathrm{N}_{2}^{+}$Meinel $(7,3)$ and $(8,4)$ and other emissions including $\mathrm{O}_{2}^{+} 1 \mathrm{~N}(2,4)$, $(1,3)$, and $(0,2)$ is at most $10-15 \%$ in the wavelength range of the filter transmission. The ASK data are calibrated using star fluxes. Measured intensities of stars are compared with theoretical tabulated values (Ivchenko et al., 2008). The uncertainty arising from this process has not been quantified for the present work. Considering all of the above sources of uncertainty, the model results of Fig. 7 show that for the cross section sets used, the variations are well within the experimental limits. 
It is necessary to consider how the field of view of ASK is filled during each time step, since there will be differences when comparing modelled and measured brightnesses if the field of view is not uniformly filled. In these observations, the time integration for the model was fixed to match that of the radar at $0.44 \mathrm{~s}$ whereas the changes in the auroral brightness in the radar field of view are faster. The images show that during the latter part of the observations there are dynamic patches which are varying in intensity on about this time scale. Modelling of such variations requires higher time resolution and attention to the spatial changes. These events have been studied in the companion paper (Lanchester et al., 2009) using cross section Set 3 for modelling $N_{2} 1 P$ emission.

\section{Conclusion}

A new auroral imager ASK provides very high temporal and spatial resolution measurements of auroral emissions from three cameras simultaneously. Measured emissions from one camera of the $\mathrm{N}_{2} 1 \mathrm{P}(4,2)$ and $(5,1)$ bands are compared with emissions resulting from model calculations. The model uses selected cross sections for excitation taken from the available literature.

The input for the model uses both optical and radar measurements. The resulting model electron density height profiles have been compared with profiles measured with the radar; the agreement is exceptionally good throughout the event. This agreement provides confirmation that the energy flux and electron energy spectra used in the model are very good estimates for the precipitation causing the emissions.

The main uncertainty in the model comes from the energy dependent cross sections for excitation to the vibrational states of the $\mathrm{N}_{2}$ molecule, and the contribution of the cascades to the B state. Cross sections for emission in the two measured bands have been constructed using different available excitation cross sections, and including all important cascades. The latter have been calculated by solving the balance equation for all sources and losses of importance. The cascade contributions account for as much as half the total emissions. The work allows us to estimate the relative importance of the various cascade contributions, including radiative transitions between the upper levels of the B state and nearby energy levels.

Within the uncertainties of the model and measurements, results of the model are in excellent agreement with the measured brightnesses during the auroral events. The constructed emission cross sections taken from the review by Itikawa (2005) make a very close match.

Future work will study the detailed changes in energy spectra for different auroral features at high spatial and temporal resolution, using the multispectral capabilities of the ASK instrument combined with modelling of all ASK emissions. Modelling of $\mathrm{N}_{2}$ emissions can be done with confi- dence that the cross sections for excitation and emission are valid.

Acknowledgements. The ASK instrument was funded by the PPARC of the UK. MA is supported by STFC. NI is supported by the Swedish Research Council (VR). We thank the EISCAT and ASK campaign teams, in particular Bjorn Gustavsson. EISCAT is an international association supported by research organisations in China (CRIRP), Finland (SA), France (CNRS, till end 2006), Germany (DFG), Japan (NIPR and STEL), Norway (NFR), Sweden (VR), and the United Kingdom (STFC).

Topical Editor M. Pinnock thanks two anonymous referees for their help in evaluating this paper.

\section{References}

Brunger, M. J. and Buckman, S. J.: Electron Molecule Scattering Cross Sections I: diatomic molecules, Phys. Rep., 357, 215-458, 2002.

Brunger, M. J. and Teubner, P. J. O.: Differential cross sections for electron-impact excitation of the electronic states of $\mathrm{N}_{2}$, Phys. Rev. A, 41, 1413-1426, 1990.

Brunger, M. J., Buckman, S., and Elford, M.: Photon and Electron Interactions with Atoms, Molecules and Ions, Landolt-Börnstein Volume I/17C, Springer-Verlag, Berlin, 2003.

Campbell, L., Brunger, M. J., Nolan, A. M., Kelly, L. J., Wedding, A. B., Harrison, J., Teubner, P. J. O., Cartwright, D. C., and McLaughlin, B.: Integral cross sections for electron impact excitation of electronic states of $\mathrm{N}_{2}$, J. Phys. B, 34, 1185-1199, 2001.

Cartwright, D. C.: Total cross sections for the excitation of the triplet states in molecular nitrogen*, Phys. Rev. A, 2, 1331-1348, 1970.

Cartwright, D. C.: Vibrational populations of the excited states of $\mathrm{N}_{2}$ under auroral conditions, J. Geophys. Res., 83, 517-531, 1978.

Cartwright, D. C., Trajmar, S., and Williams, W.: Vibrational population of the $A^{3} \Sigma_{\mathrm{u}}^{+}$and $B^{3} \Pi_{\mathrm{g}}$ states of $\mathrm{N}_{2}$ in normal auroras, J. Geophys. Res., 76, 8368-8377, 1971.

Cartwright, D. C., Trajmar, S., Chutjian, A., and Williams, W.: Electron impact excitation of the electronic states of $\mathrm{N}_{2}-$ II. Integral cross sections at incident energies from 10 to $50 \mathrm{eV}$, Phys. Rev. A, 16, 1041-1051, 1977a.

Cartwright, D. C., Trajmar, S., Chutjian, A., and Williams, W.: Electron impact excitation of the electronic states of $\mathrm{N}_{2}-\mathrm{I}$. Differential cross sections at incident energies from 10 to $50 \mathrm{eV}$, Phys. Rev. A, 16, 1013-1040, 1977b.

Chung, S. and Lin, C. C.: Excitation of the electronic states of the nitrogen molecule by electron impact, Phys. Rev. A, 6, 9881002, 1972.

Gattinger, R. L. and Vallance-Jones, A.: Quantitative spectroscopy of the aurora - II. The spectrum of medium intensity aurora between 4500 and $8900 \AA$, Can. J. Phys, 52, 2343-2356, 1974.

Gattinger, R. L., Vallance Jones, A., Hecht, J. H., Strickland, D. J., and Kelly, J.: Comparison of ground-based optical observations of $\mathrm{N}_{2}$ second positive to $\mathrm{N}_{2}(+)$ first negative emission ratios with electron precipitation energies inferred from the Sondre Stromfjord radar, J. Geophys. Res., 96, 11341-11351, 1991. 
Gillan, C. J., Tennyson, J., McLaughlin, B. M., and Burke, P. G.: Low-energy electron impact excitation of the nitrogen molecule: optically forbidden transitions, J. Phys. B, 29, 1531-1547, 1996.

Gilmore, F. A.: Comment on paper 'semi-empirical electron impact cross sections for atmospheric gases', Can. J. Chem., 47, 1779, 1969.

Gilmore, F. R., Laher, R. R., and Epsy, P. J.: Franck-Condon factors, r-centroids, electronic transition moments, and Einstein coefficients for many nitrogen and oxygen band systems, J. Phys. Chem. Ref. Data, 21, 1005-1106, 1992.

Hedin, A. E.: Extension of the MSIS Thermospheric Model into the Middle and Lower Atmosphere, J. Geophys. Res., 96, 11591172, 1991.

Itikawa, Y.: Cross Sections for Electron Collisions with Nitrogen Molecules, J. Phys. Chem. Ref. Data, 35, 31-53, 2005.

Johnson, P. V., Malone, C. P., Kanik, I., Tran, K., and Khakoo, M. A.: Integral cross sections for the direct excitation of the $A^{3} \Sigma_{\mathrm{u}}^{+}, B^{3} \Pi_{\mathrm{g}}, W^{3} \Delta_{\mathrm{u}}, B^{\prime}{ }^{3} \Sigma_{\mathrm{u}}^{-}, a^{\prime}{ }^{1} \Sigma_{\mathrm{u}}^{-}, a^{1} \Pi_{\mathrm{g}}, w^{1} \Delta_{\mathrm{u}}$, and $C^{3} \Pi_{\mathrm{u}}$ electronic states in $\mathrm{N}_{2}$ by electron impact, J. Geophys. Res., 110, A11311, doi:10.1029/2005JA011295, 2005.

Jokiaho, O., Lanchester, B. S., Ivchenko, N., Daniell, G. J., Miller, L. C. H., and Lummerzheim, D.: Rotational temperature of $\mathrm{N}_{2}^{+}$ $(0,2)$ ions from spectrographic measurements used to infer the energy of precipitation in different auroral forms and compared with radar measurements, Ann. Geophys., 26, 853-866, 2008, http://www.ann-geophys.net/26/853/2008/.

Khakoo, M. A., Johnson, P. V., Ozkay, I., Yan, P., Trajmar, S., and Kanik, I.: Differential cross sections for the electron impact excitation of the $A^{3} \Sigma_{\mathrm{u}}^{+}, B^{3} \Pi_{\mathrm{g}}, W^{3} \Delta_{\mathrm{u}}, B^{\prime}{ }^{3} \Sigma_{\mathrm{u}}^{-}, a^{\prime}{ }^{1} \Sigma_{\mathrm{u}}^{-}$, $a^{1} \Pi_{\mathrm{g}}, w^{1} \Delta_{\mathrm{u}}, C^{3} \Pi_{\mathrm{u}}$ states of $\mathrm{N}_{2}$, Phys. Rev. A, 71, 062703, doi:10.1103/PhysRevA.71.062703, 2005.

Lanchester, B. S., Rees, M. H., Lummerzheim, D., Otto, A., Frey, H. U., and Kaila, K. U.: Large fluxes of auroral electrons in filaments of 100 m width, J. Geophys. Res., 102, 9741-9748, 1997.

Lanchester, B. S., Ashrafi, M., and Ivchenko, N.: Simultaneous imaging of aurora on small scale in $\mathrm{OI}(777.4 \mathrm{~nm})$ and $\mathrm{N}_{2} 1 \mathrm{P}$ to estimate energy and flux of precipitation, Ann. Geophys., submitted, 2009.

Lummerzheim, D.: Electron transport and optical emissions in the aurora, $\mathrm{PhD}$ thesis, Univ. of Alaska, Faribanks, 1987.
Lummerzheim, D. and Lilensten, J.: Electron transport and energy degradation in the ionosphere: evaluation of the numerical solution, comparison with laboratory experiments and auroral observations, Ann. Geophys., 12, 1039-1051, 1994, http://www.ann-geophys.net/12/1039/1994/.

Majeed, T. and Strickland, D. J.: New survey of electron impact cross sections for photoelectron and auroral electron energy loss calculations, J. Phys. Chem. Ref. Data, 26, 335-349, 1997.

McConkey, J. W. and Simpson, F. R.: Electron impact excitation of the $\mathrm{B}^{3} \Pi_{\mathrm{g}}$ stae of $\mathrm{N}_{2}$, J. Phys. B, 2, 923-929, 1969.

Morrill, J. S. and Benesch, W. M.: Auroral $\mathrm{N}_{2}$ emissions and the effect of collisional processes on $\mathrm{N}_{2}$ triplet state vibrational populations, J. Geophys. Res., 101, 261-274, 1996.

Ohmori, Y., Shimozuma, M., and Tagashira, H.: Boltzmann equation analysis of electron swarm behaviour in nitrogen, J. Phys. D: Appl. Phys., 21, 724-729, 1988.

Shemansky, D. E. and Broadfoot, A. L.: Excitation of $\mathrm{N}_{2}$ and $\mathrm{N}_{2}^{+}$ systems by electrons - II. Excitation cross sections and $\mathrm{N}_{2} 1 \mathrm{PG}$ low pressure afterglow, J. Quant. Spectrosc. Radiat. Transf., 11, 1401-1437, 1971.

Shemansky, D. E. and Broadfoot, A. L.: Comment on paper by D. C. Cartwright, S. Trajmar and W. Williams, Vibrational population of the $A^{3} \Sigma_{\mathrm{u}}^{+}$and $B^{3} \Pi_{\mathrm{g}}$ states of $\mathrm{N}_{2}$ in normal auroras, J. Geophys. Res., 78, 2357-2364, 1973.

Stamnes, K. and Rees, M. H.: Heating of thermal ionospheric electrons by superthermal electrons, Geophys. Res. Lett., 10, 309312, 1983.

Stanton, P. N. and St. John, R. M.: Electron excitation of the first positive bands of $\mathrm{N}_{2}$ and of the first negative and Meinel bands of $\mathrm{N}_{2}^{+}$, J. Opt. Soc. Am., 59, 252-260, 1969.

Tashiro, M. and Morokuma, K.: R-matrix calculation of integral and differential cross sections for low-energy electron-impact excitations of the $\mathrm{N}_{2}$ molecule, Phys. Rev. A, 75, 012720, doi:10.1103/PhysRevA.75.012720, 2007.

Trajmar, S., Register, D. F., and Chutjian, A.: Electron scattering by molecules. II - Experimental methods and data, Phys. Rep., 97, 219-356, 1983.

Vallance-Jones, A.: Aurora, Cambridge University Press, 1974.

Wu, H. L. and Benesch, W.: Evidence for the ${ }^{3} \Delta_{\mathrm{u}} \rightarrow \mathrm{B}^{3} \Pi_{\mathrm{g}}$ : Transition in $\mathrm{N}_{2}$, Phys. Rev., 172, 31-35, 1968. 